\title{
Fermented Prebiotic Dietary Supplement AferBio
}

National Cancer Institute

\section{Source}

National Cancer Institute. Fermented Prebiotic Dietary Supplement AferBio. NCI

Thesaurus. Code C156736.

A powder-based nutritional supplement composed of Brazilian fermented non-digestible food composed of soy, wheat, and organic wheat germ, and containing a mixture of non-soluble and fermentable fibers rich in oligosaccharides, with potential immunomodulating and prebiotic activities. Upon oral administration of the fermented prebiotic dietary supplement, the high concentrations of beta-glucans are able to stimulate the proliferation of beneficial bacteria in the gastrointestinal (GI) tract. This modulates the Gl flora ecosystem, improves the functions of the intestinal barrier and protects against harmful viruses and bacteria. In addition, this prebiotic modulates the immune system and may inhibit inflammation. 